\title{
Erfolgreiche Entwicklung der Ärztenetze in der Schweiz
}

Peter Berchtold, Karen Peier, Christian Peier

Korrespondenz:

PD Dr. med. Peter Berchtold

College-M

Freiburgstrasse 41

CH-3010 Bern

peter.berchtold@college-m.ch
Die Entwicklung der Ärztenetze in der Schweiz ist eine Erfolgsgeschichte: Jahr für Jahr entscheiden sich mehr Versicherte für entsprechende Versicherungsprodukte, mehr Patienten für eine verbindliche, hausärztlich koordinierte Behandlung und mehr (Haus-)Ärztinnen und Ärzte für eine institutionalisierte Zusammenarbeit in Ärztenetzen. Bedeutsam ist, dass diese Entwicklungen in vielen Teilen durch die Ärzte selbst initialisiert und vorangetrieben wurden und werden und von der Standesorganisation nicht nur unterstützt, sondern aktiv mitgestaltet werden [1]. Das ist ein wesentliches Unterscheidungsmerkmal zur Entwicklung von Managed Care in anderen mitteleuropäischen Ländern, die deutlich weniger durch das Kerngeschäft getragen bzw. sogar bekämpft wird.

\section{Eine nationale Erhebung}

Doch welches ist der Entwicklungsstand der Ärztenetze in der Schweiz und welche regionalen und weiteren Unterschiede gibt es? Diese Fragen sind Kern einer nationalen Erhebung, die College-M im Auftrag des Forums Managed Care seit einigen Jahren durchführt. Diese jährliche Studie umfasst 1. quantitative Daten wie Anzahl und Grösse (Versicherte, Ärzte) der Netze pro Kanton und 2. eine wachsende Anzahl qualitativer Charakteristika wie Qualitätsmassnahmen oder Budgetmitverantwortung. Die Angaben werden von sämtlichen Ärztenetzen in der Schweiz bzw. deren Betriebsgesellschaften mittels eines Fragebogens und Direktansprachen erhoben, d.h. diese Erhebung umfasst alle uns bekannten Ärztenetze in der Schweiz. Die vorliegende Publikation fasst die wichtigsten Resultate der Erhebung 2008 zusammen. Die Daten zu den einzelnen Ärztenetzen werden zeitgleich in der Zeitschrift «Managed Care» [2] bzw. auf der Webseite des Forum Managed Care [3] publiziert.

Wir zählen aktuell 86 Ärztenetze in der Schweiz. Ärztenetze definieren wir als Organisationen, die durch vertraglich vereinbartes Zusammenwirken unter sich, mit netzfremden Leistungserbringern und mit den Kostenträgern auf die Bedürfnisse der Patientinnen und Patienten ausgerichtete Gesundheitsleistungen erbringen.
Dieses Zusammenwirken beruht unter anderem auf vereinbarten Behandlungsprozessen, unternehmerischen Organisationsstrukturen, effektivem Umgang mit den vorhandenen Mitteln, gemeinsamer Betreuungskultur und dem Willen, die Gesundheitsleistungen aus einer (hausärztlichen) Hand zu steuern [4].

\section{Die Netze unterscheiden sich}

Die 86 Ärztenetze in der Schweiz unterscheiden sich in vielerlei Hinsicht: Abbildung 1 zeigt die geographische Verteilung der Ärztenetze, deren Grösse - Anzahl der im Netz betreuten Versicherten und angeschlossenen Ärzte - sowie das Netzalter nach Betriebsjahren. Zu beachten ist die Häufung der Netze in den nordostschweizerischen Regionen sowie den Kantonen Bern, Genf und Luzern. Ein ähnliches Bild ergibt sich für den prozentualen Anteil der Versicherten bzw. der ärztlichen Grundversorger pro Kanton (Abb. 2): Keinen Zusammenschluss von Ärzten in Netzwerken und keine entsprechenden Versicherungsprodukte (Hausarztmodelle u.a.) finden wir in den Kantonen Glarus, Jura, Neuenburg, Obwalden, Waadt und Wallis. Nur geringe Aktivitäten betreffend Anteil der Versicherten in Ärztenetzen $(<10 \%)$ finden wir in den beiden Appenzeller Halbkantonen sowie in den Kantonen Freiburg, Nidwalden, Solothurn, Schwyz, Tessin, Uri und Zug. Ärzteseitig unbedeutend, d.h. $<10 \%$ der Grundversorger sind Ärztenetzen angeschlossen, scheinen die Kantone Uri und Tessin.

\section{Struktur und Qualitätsmerkmale}

Beinahe alle Netze haben eine oder mehrere Formen eines netzweiten Qualitätsmanagements implementiert (Tab. 1): Schwerpunkt sind die Qualitätszirkel, die 99\% der Netze verbindlich durchführen. Der Medianwert der obligatorischen Zirkel liegt bei 8 pro Jahr. Weitere Qualitätsmerkmale sind Behandlungsleitlinien, institutionalisierte Patienteninformationen, Ombudsstelle und Patientenrat. Ein netzeigener Notfalldienst wird von $63 \%$, ein telefonischer Beratungsdienst von $52 \%$ der Netze angeboten.

Eine Budgetmitverantwortung (BMV) ist zu einem wesentlichen Element der Schweizer Ärzte- 


\section{Abbildung 1}

Ärztenetze mit der Anzahl der im Netz betreuten Versicherten $(\square)$ bzw. angeschlossenen Ärzte $(\square)$.

Grafik: Hahn und Zimmermann, Bern.

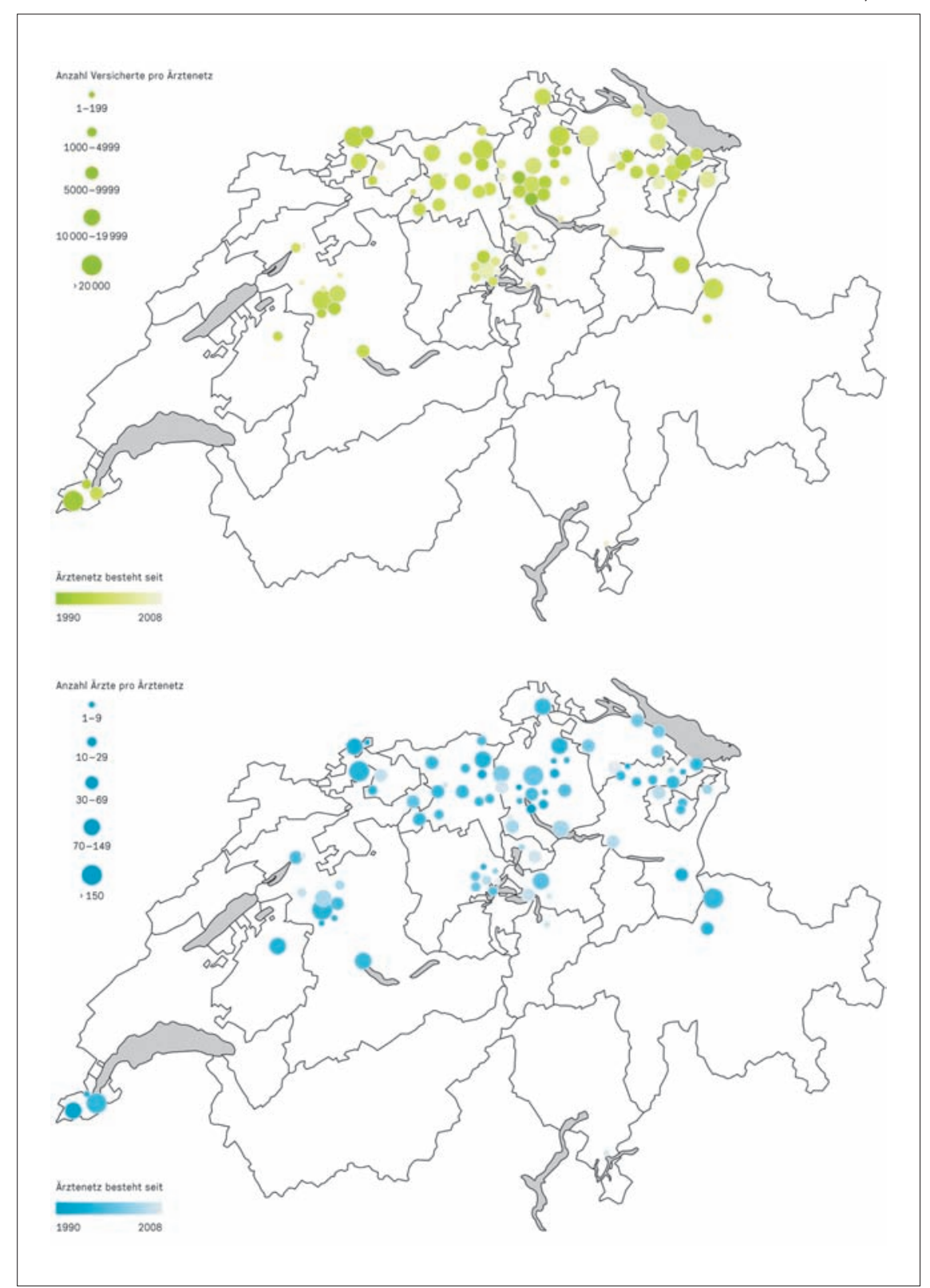

netze geworden. BMV meint die Verantwortung des Ärztenetzes (und nicht des einzelnen Arztes!), für eine bestimmte Gruppe von Patienten ein vereinbartes Kostenziel einzuhalten [5]. Gerade von ärztlicher Seite wird die Budgetmitverantwortung als «ein erfolgversprechendes Zusammenarbeitsprinzip zwischen Ärztenetzwerken und Versicherern und als eine zukunftsweisende Chance für die Ärzteschaft, das Gesundheitssystem mitzugestalten» gesehen [6]. Abbildung 3 zeigt die einzelnen Ärztenetze pro Kanton in Abhängigkeit der Anzahl angeschlossener Ärzte (blaue Achse), der Anzahl betreuter Versicherter (grüne Achse) sowie der Budgetmitverantwortung (farbige Fläche mit BMV/graue Fläche ohne BMV). Beachtenswert ist, dass jene Kantone mit langjährig bestehenden Netzen (z. B. ZH, AG, SG, TG) fast ausschliesslich solche mit BMV haben, während Kantone mit jüngeren Netzen (z. B. BE, GE, GR) eher solche ohne BMV aufweisen.

\section{Fazit}

Gegenüber 2004 hat die Anzahl der in Ärztenetzen betreuten Versicherten um 34\% und die Anzahl der in Netzen zusammengeschlossenen Grundversorger um 17\% zugenommen [7]. Dies ist eine überaus erfolgreiche Entwicklung, wenn man bedenkt, dass diese Entwicklung in erster Linie durch Netze sowie Kassen/Versicherer bzw. durch diese gemeinsam vorangetrieben und nicht - wie in anderen Ländern üblich - durch staatlichen Interventionismus oder sogenannte Anschubfinanzierungen provoziert wurde. Die Hypothese gilt, dass gerade darin, d.h. in der Freiwilligkeit und den Experimentiermöglichkeiten, ein wesentlicher Erfolgsfaktor der Schweizer Managed-Care-Entwicklung liegt [1]. Diese Entwicklung findet aber nicht nur als Grössenwachstum statt, auch die (vertragliche) Verbindlichkeit der Ärztenetze nimmt zu. Als Mass dafür kann die Budgetmitverantwortung der Netze herangezogen werden: Während 2007 von 81 Netzen erst $62 \%$ eine Form der Budgetmitverantwortung (Bonus-Malus-Systeme oder Capitation) mit den Kassen vereinbart hatten [8], waren es in diesem Jahr bereits $79 \%$ der Netze.

Die vorliegende Übersicht erhebt zwar den Anspruch auf Vollständigkeit und ist gleichzeitig eine momentane Bestandsaufnahme, die die grosse Dynamik der Netzaktivitäten in der ambulanten Versorgung ungenügend abbildet: Bereits heute zählen wir mindestens 8 neue Ärztenetze im Aufbau sowie viele weitere Initiativen. Diese Initiativen zielen in Richtung Weiterentwicklung der Netzorganisationen, Intensivierung der Qualitätssysteme und Erweiterung des GatekeepingPrinzips insbesondere bei chronischen Krankheiten. Diese Entwicklungen sollen in der nächsten Erhebung 2010 erfasst werden. 
Abbildung 2

Anteil der Versicherten ( $\square$ ) bzw. ärztlichen Grundversorger ( $\square$ ) in Ärztenetzen pro Kanton.

Grafik: Hahn und Zimmermann, Bern.

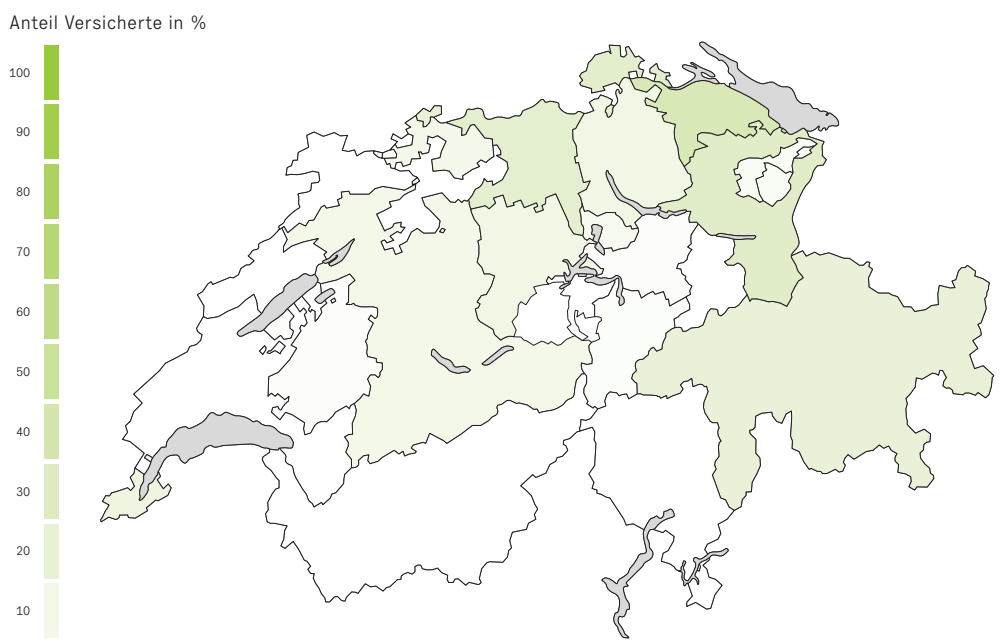

Anteil Grundversorger in \%

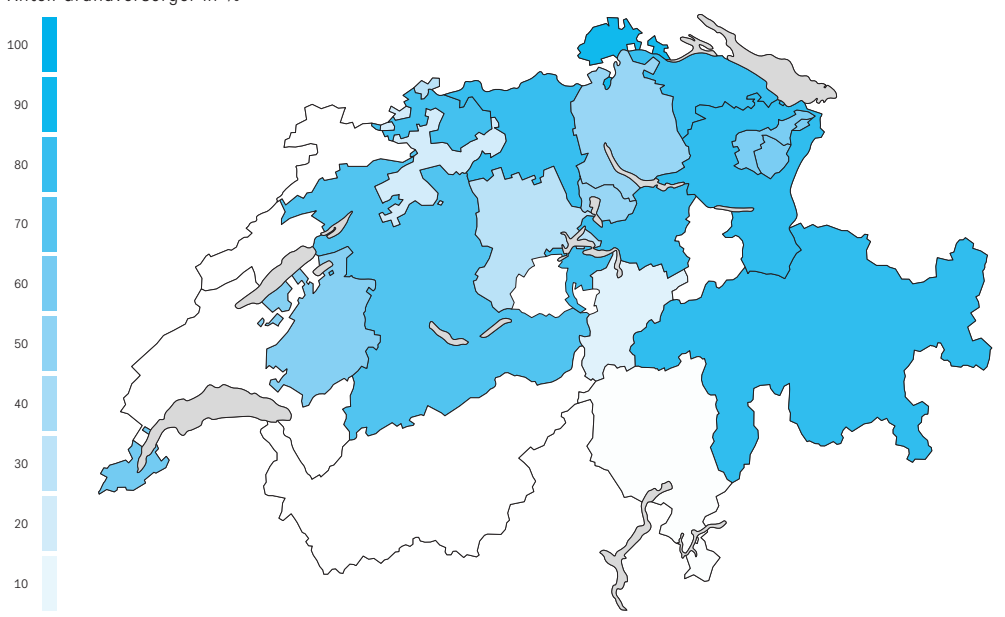

\section{Literatur}

1 AGMC-FMH. Thesenpapier FMH. Managed Care Kontrahierungszwang. Schweiz Ärztezeitung. 2006;87(36):536-40.

2 Peier K, Peier C, Berchtold P. Ärztenetze in der Schweiz: Übersicht und Merkmale 2008. Care Management. 2008;§§: in press.

3 www.fmc.ch.

4 Definition Ärztenetze gemäss med-swiss.net, Dachverband der Schweizer Ärztenetzwerke. www.medswiss.net/projekte/dateien/\%c4rztenetzwerke_vonVision-zur-Realit\%e4t_V5.pdf.

5 Götschi AS, Weber A. Ein Budget für Ärzte? Alles, was Sie schon immer über Budgetverantwortung wissen wollten. Schweiz Ärztezeitung. 2004;85(47):2498-503.

6 Dreiding P, Fritschi J. Warum Begriffe aus der Ökonomie uns Ärztinnen und Ärzte nicht erschrecken müssen! Schweiz Ärztezeitung. 2006;87(30):1315-9.

7 Münger I, Berchtold P, Hess K. Managed-Care-Modelle in der Schweiz. Managed Care. 2004;7:35-9.

8 Berchtold P, Hess K. Ärztenetze in der Schweiz. Managed Care. 2007;6:33-40.

\section{Tabelle 1}

Struktur- und Qualitätsmerkmale der Ärztenetze (n/\% Netze).

\begin{tabular}{|lllllll} 
Patientenrat & Ombudsstelle & Qualitätszirkel & Guidelines & Patienteninformation & Notfalldienst & Beratungstelefon \\
\hline 2 & 36 & 85 & 51 & 46 & 54 & 45 \\
$2 \%$ & $42 \%$ & $99 \%$ & $59 \%$ & $53 \%$ & $63 \%$ & $52 \%$
\end{tabular}




\section{Abbildung 3}

Vergleichende Darstellung der Netze pro Kanton in Abhängigkeit der angeschlossenen Ärzte ( $\square$ ), der betreuten Versicherten ( $\square$ ) und der Budgetmitverantwortung ( Netz mit Budgetmitverantwortung / N Netz ohne Budgetmitverantwortung).

Grafik: Hahn und Zimmermann, Bern.

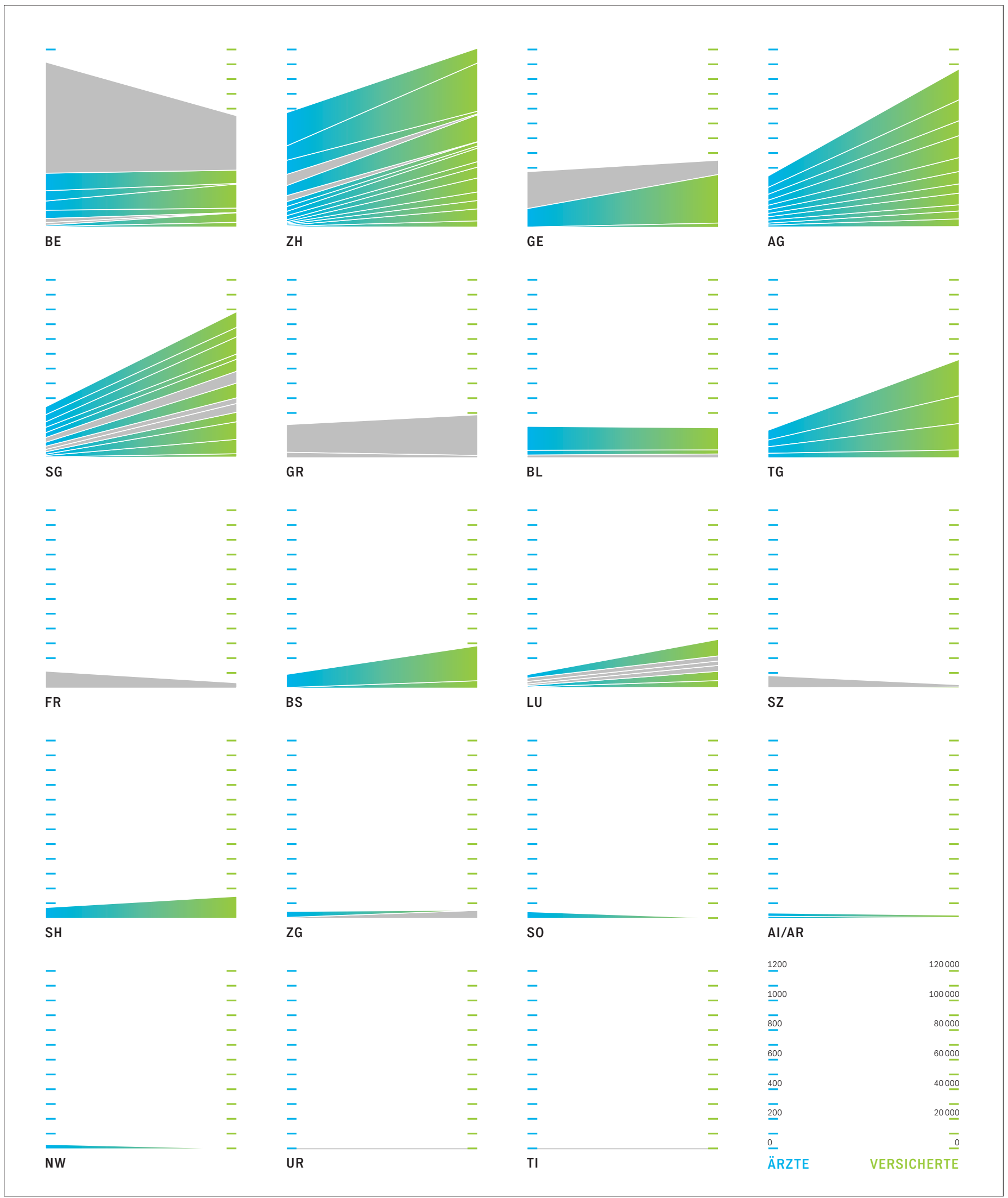

